of seasoning methods and treatment of the reactor. Traces of high boiling impurities in the reactant accelerated the reaction rate. Small amounts of polymer appeared in the products, and some samples, heated for long periods, showed the presence of chlorine and hydrogen chloride. The rate constants in a given run under our better conditions showed deviations from the average of from 0 to $40 \%$. A few runs carried to $40 \%$ to $50 \%$ cis-production displayed a systematic rise in rate, as expected from our previous remarks. Our best procedure for treatment of the reactor gave rate constants reproducible to within $\sim 50 \%$.

Jones and Taylor experienced difficulty with surface effects but appeared to have finally obtained reproducible, homogeneous conditions. Nonetheless, their data for the trans-isomer reveals evidence of irreproducibility and inconsistency. Tamamushi et al. claimed homogeneous reaction but produced no data bearing on this point, nor is the precision of their rate constants good. The rate constants found by us were from 50 to 150 times smaller than those of Jones and Taylor at comparable temperatures, while our rates at $275^{\circ} \mathrm{C}$ were already tenfold less than those of Tamamushi et al. at $250^{\circ} \mathrm{C}$. Since there is no relevant manner in which an elementary, unimolecular isomerization reaction could have had its rate depressed below the true value, we conclude that there is no reliable rate data available on the homogeneous, unsensitized cis-trans isomerization of dichloroethylene, and there is no evidence to suggest that it is an exceptional example of a unimolecular reaction.

* This work was supported by the National Science Foundation. $\dagger$ Fulbright Exchange Fellow.

1 J. L. Jones and R. L. Taylor, J. Am. Chem. Soc. 62, 3480 (1940).

2 Tamamushi, Akiyama, and Ishii, Z. Elektrochem. 47, 340 (1941).

3 (a) Magee, Shand, and Eyring, J. Am. Chem. Soc. 63, 677 (1941); (b) Glasstone, Laidler, and Eyring, The Theory of Rate Processes (McGraw-Hill Book Company, Inc., New York, 1941), pp. 331, 611 .

\section{EPR Spectrum of Solid Nitrogen Afterglow at $4.2^{\circ} \mathrm{K}$}

T. Cole,, John T. Harding, $†$ John R. Pellam, AND DoN M. YosT

Gates and Crellin Laboratories of Chemistry and Norman Bridge Laboratory of Physics, California Institute of Technology, Pasadena, California

(Received May 24, 1957)

$B^{\mathrm{r}}$ ECAUSE the Rayleigh afterglow induced in nitrogen is of undoubted importance to physics, chemistry, and astrophysics, as well as in studies of the earth's atmosphere, it seemed of primary interest to determine whether thermally condensed activated nitro-

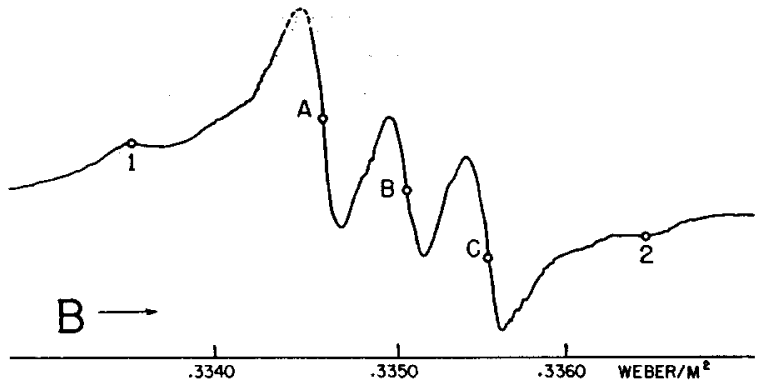

FIG. 1. EPR spectrum of atomic nitrogen condensed at $4.2^{\circ} \mathrm{K}$. Frequency $9397 \mathrm{mc}$.

gen would yield an electron paramagnetic resonance (EPR) spectrum.

We have recently observed intense EPR spectra in the afterglow solidified at $4.2^{\circ} \mathrm{K}$, and they plainly indicate the presence of atomic nitrogen.

Afterglow was produced by conducting nitrogen gas at a pressure of about $1 \mathrm{~mm} \mathrm{Hg}$ through a microwave resonator connected to a 2450 -mc Raytheon diathermy device. ${ }^{1}$ The pressure was so adjusted that the familiar yellow afterglow was observed; the glowing gas was then condensed on the walls of the $x$-band sample cavity at $4.2^{\circ} \mathrm{K}$ in the form of a glowing solid ${ }^{2,3}$; the glow was now green in color.

The EPR spectrograph, of our own construction, incorporates an A. D. Little electromagnet which we have equipped with an automatic field control and sweep governed by a proton resonance fluxmeter. A $2 \mathrm{~K} 25$ klystron with automatic frequency control provided microwave radiation at a frequency of $9397 \mathrm{mc}$. The sample cavity was a simple rectangular reflection cavity operating in the $T E_{011}$ mode. A balanced "magic" tee bridge was used, one of whose $\mathrm{H}$ arms extended into a Dewar filled with liquid helium and was terminated by the sample cavity. Magnetic field modulation of $10^{-4}$ weber $/ \mathrm{m}^{2}$ (1 gauss) at $400 \mathrm{cps}$ allowed the resonance signal to be received by a phase sensitive detector, thus producing the derivative of the absorption curve.

Figure 1 shows one of the spectra obtained; it consists of three distinct lines whose estimated center values are marked $A, B$, and $C$. The splittings from the center line of the triplet are $4.5 \pm 0.4 \times 10^{-4}$ weber $/ \mathrm{m}^{2}$. The value of $g_{j}$ for the center line is $2.0005 \pm 0.0002$ and indicates an atom in an $S$ state. Two weak satellite lines may also be noticed at points 1 and 2 ; their separations from $B$ are 14 and $16 \times 10^{-4}$ weber $/ \mathrm{m}^{2}$, respectively. There was no noticeable decrease in signal after one hour, thus denoting a long half-life for the solid active nitrogen.

In its ground state, very nearly $1 s^{2} 2 s^{2} 2 p^{3}{ }^{4} S_{3}$, atomic nitrogen has a total electronic spin of $S=\frac{3}{2}$ and a nuclear spin of $I=1$. As Hamiltonian for an atom in an $S$ state in a magnetic field we take,

$$
\mathbf{H}=-\hbar\left(\gamma_{1} \mathbf{S}+\gamma_{2} \mathbf{I}\right) \cdot \mathbf{B}+A \mathbf{I} \cdot \mathbf{S},
$$


where $\gamma_{1}$ and $\gamma_{2}$ are the magnetogyric ratios of the electronic and nuclear systems, respectively; $\mathbf{S}$ and $\mathbf{I}$ are the angular momentum operators for the electronic and nuclear systems; $A$ is the hyperfine coupling constant. First-order perturbations result in three allowed paramagnetic transitions, viz.,

$$
h \nu=\hbar \gamma_{1} B, \quad \hbar \gamma_{1} B \pm A \hbar^{2},
$$

which correspond to the three strong lines observed.

Analysis of the data yields a value of $A=4.5 \times 10^{-4}$ weber $/ \mathrm{m}^{2}$, or $A=12.6 \mathrm{mc}$, as compared with $10.45 \mathrm{mc}$ found by Heald and Beringer ${ }^{4}$ for the magnetic resonance of nitrogen afterglow at room temperature. Second-order perturbation leads to additional splitting which, if unresolved, produces a shift of the center of gravity of the spectrum relative to the free electron line of the order of $\frac{1}{4}\left(A^{2} \hbar^{3} / \gamma_{1} B\right)$. For the above value of $A$ and a field of 0.33 weber $/ \mathrm{m}^{2}$, which we employed, this shift is $1.5 \times 10^{-7}$ weber $/ \mathrm{m}^{2}$, far below our limit of resolution. In the case of hydrogen and deuterium where $A$ is much greater, owing to the large value of $\psi^{2}(0)$ for $1 S$ orbits, second-order terms account quantitatively for the shift of $g_{j}$ values in these atoms as reported by Jen $e t a l .{ }^{5}$

No explanation for the two weak satellite lines 1 and 2 nor for the difference in coupling constant between room temperature and $4.2^{\circ} \mathrm{K}$ is offered. Further experiments are in progress. We wish to thank Dr. H. M. McConnell, of this Institute, and Dr. Floyd B. Humphrey for their many helpful discussions. This research was supported in part by the Newmont Fund (DMY), and by the Sloan Foundation and the National Science Foundation (JRP).

* U. S. Rubber Company Fellow.

+ Standard Oil Company of California Fellow.

A. M. Bass and H. P. Broida, Phys. Rev. 101, 1740 (1956).

2 J. C. McLennan and C. M. Shrum, Proc. Roy. Soc. (London) A106, 138 (1924).

${ }^{3}$ H. P. Broida and J. R. Pellam, Phys. Rev. 95, 845 (1954).

${ }^{4}$ Heald and Beringer, Phys. Rev. 96, 645 (1954).

5 Jen, Foner, Cochran, and Bowers, Phys. Rev. 104, 846 (1956).

\section{Magnetic Susceptibility of $\mathrm{NpF}_{6} \dagger$}

Bernard Weinstock and John G. Malm Argonne National Laboratory, Lemont, Illinois

(Received June 17, 1957)

$T$ HE small temperature-independent paramagnetic susceptibility observed for $\mathrm{PuF}_{6}$ has been explained on the basis that its two nonbonding electrons occupy a nondegenerate ground state with paired spins, this state being formed by the splitting of an $f$ term in the octahedral field of the molecule. ${ }^{1}$ By contrast, other actinide compounds that contain two nonbonding electrons in compounds of different configurational symmetry show large temperature dependent suscep-

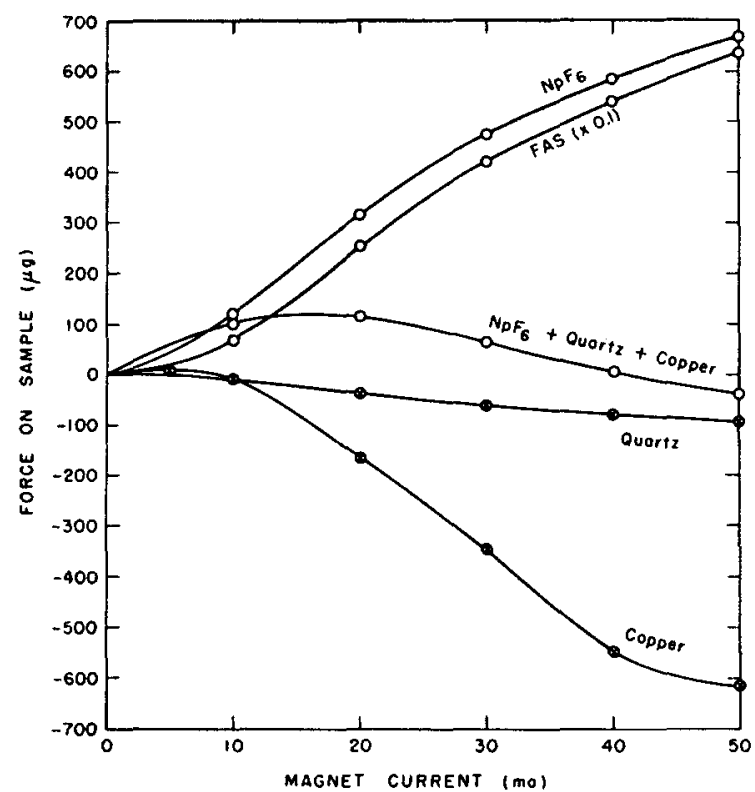

FIG. 1. Magnetic force measurements at $294.9^{\circ} \mathrm{K}$ as a function of magnet current for the empty copper and quartz containers and for the assembly with $\mathrm{NpF}_{6}$. The calculated net forces on the $\mathrm{NpF}_{6}$ alone and one-tenth of the calculated net forces on the Mohr's salt (FAS) sample used for calibration are also shown.

tibilities. $^{2-4}$ In view of this interesting difference we have extended our studies to the magnetic susceptibility of $\mathrm{NpF}_{6}$, which contains a single nonbonding electron in a molecule of octahedral symmetry. ${ }^{5}$

The measurements were made by the Faraday method with apparatus and technique similar to that used for $\mathrm{PuF}_{6} .{ }^{1}$ Polycrystalline $\mathrm{NpF}_{6}(47.4 \mathrm{mg})$ sealed in a 4-mm quartz sphere and enclosed in a copper capsule was located between the poles (isodynamic gap type) ${ }^{6}$ of an electromagnet by a gold chain suspended from a microbalance. Determinations of the weight change upon application of the magnetic field were then made at several magnet currents and at temperatures of $64.0,77.2$, and $294.9^{\circ} \mathrm{K}$ for the empty quartz and copper containers as well as for the assembly containing the $\mathrm{NpF}_{6}$. The observed weight changes were converted into susceptibilities by calibration with Mohr's salt, ${ }^{7}$ the susceptibility being taken as proportional to the observed weight change. The values obtained for the susceptibility of $\mathrm{NpF}_{6}$ varied with magnetic field, indicating a small ferromagnetic impurity. This effect saturated at the smallest field used and was corrected for by subtracting a constant force $(86 \mu \mathrm{g})$ from the forces observed at larger fields. ${ }^{8}$ The observed forces at room temperature on the empty copper and quartz containers and on the $\mathrm{NpF}_{6}$ assembly as a function of magnet current are shown in Fig. 1. Also plotted are the net forces on the $\mathrm{NpF}_{6}$ alone and one-tenth of the net forces on the calibration sample. The molar susceptibilities of $\mathrm{NpF}_{6}$ are given in Table I as well as values obtained from measurements taken a month after filling. The small increase in susceptibility at this later 